\title{
NUEVOS MODELOS PARA EL RESCATE DEL CAPITAL HUMANO. EL ACUERDO 286
}

\author{
José O. Medel Bello \\ Osvaldo Edmundo Escalante Jarero
}

\section{RESUMEN}

Hoy, el conocimiento y la información son algunos de los principales activos con que cuentan las naciones. El desarrollo de estrategias ágiles e innovadoras que permitan reconocer y recuperar la experiencia, conocimientos y habilidades de quienes han realizado esfuerzos valiosos en su formación -tanto intelectual como laboral-, posibilita rescatar el capital humano e incrementa la competitividad profesional de la sociedad. El Acuerdo secretarial número 286, de la Secretaría de Educación Pública, es una innovación en materia educativa; permite, a todas aquellas personas que hayan obtenido conocimientos de forma autodidacta o a través de la experiencia laboral, acreditar formalmente aquellos que correspondan a cierto nivel educativo o grado escolar.

\section{INTRODUCCIÓN}

En el contexto de globalización, el cúmulo de conocimiento es uno de los principales activos de las naciones. El acervo colectivo de conocimientos que posee un pueblo es un elemento estratégico para posibilitar el desarrollo y la movilidad social.

Las nuevas estructuras de organización social facilitan que un mayor número de personas asciendan en la pirámide educativa, favoreciéndose la igualdad de oportunidades en materia 
de educación, a fin de propiciar la generación de terrenos favorables para la edificación de proyectos sociales justos y equitativos.

El desarrollo de estrategias innovadoras que reconozcan y recuperen la experiencia, conocimientos y habilidades de quienes han realizado esfuerzos valiosos en su formación -intelectual y laboralmente-, pero que por diversas situaciones no han podido acceder o continuar con su educación escolarizada, es una de las acciones necesarias a fin de obtener un mejor nivel de vida.

Contar con programas que permitan rescatar el capital humano, disperso en los individuos que se encuentran subvaluados y desaprovechados en sus centros laborales (por no poseer una certificación formal de sus estudios, pese a contar con el conjunto de conocimientos, habilidades, destrezas, aptitudes y valores necesarios para responder a los requerimientos de las empresas), es imprescindible a fin de ampliar las capacidades laborales de la población e incrementar la competitividad profesional de la sociedad.

México, país marcado por grandes desigualdades dentro de la sociedad -manifestadas en aspectos como la dispar capacidad económica entre los individuos, las diferentes oportunidades de acceso a la educación, la desigual capacidad alimentaria, entre otras-, requiere y obliga a la sociedad en su conjunto a buscar la equidad social y educativa, y compromete a que se resuelvan los rezagos, a fin de posibilitar la futura prosperidad del país. Todo ello como elemento básico para revisar y reorganizar el referente al ámbito educativo, con la intención de lograr satisfacer las necesidades nacionales.

Ofrecer oportunidades educativas a toda la población; diversificar las opciones de educación tanto formal como no formal; aumentar el número de alumnos graduados de cada uno de los distintos niveles educativos; introducir las innovaciones necesarias que posibiliten mejorar la calidad, la pertinencia y la equidad, son acciones indispensables en materia 
educativa que permitirán establecer, de antemano, las condiciones para el pleno aprovechamiento del patrimonio humano con que cuenta el país.

Dado que todos los tipos, niveles y modalidades educativas son importantes (pues responden a necesidades y aspiraciones individuales y sociales), el combatir el rezago educativo, se hace no sólo necesario sino que se convierte en un principio de justicia social, en países como México -donde el grado promedio de escolaridad de la población de 15 años y más es de 7.6 años ${ }^{1}$; y donde, con relación al total de la población que en el año de 1999 ingresó a cursar estudios de nivel licenciatura, menos del 35\% se tituló ${ }^{2}-$.

Una de las vías a fin de enfrentar este rezago, involucra el introducir estrategias y acciones innovadoras en materia de educación, flexibilizando sus sistemas y programas, desarrollando mecanismos de normalización y reconocimiento de aprendizajes empíricos y competencias laborales.

Gracias a la necesidad de atender a la población, que por diversas causas no ha obtenido resultados favorables en la educación formal, surgen procesos como el Acuerdo secretarial número 286, que reconoce el auto-didactismo y la experiencia laboral, brindando la oportunidad de acreditar oficialmente los conocimientos equivalentes a los de cierto nivel educativo o grado escolar, por medio de certificados o títulos, permitiendo incrementar y rebasar la educación recibida.

\section{CARACTERÍSTICAS GENERALES DEL ACUERDO 286}

El Acuerdo 286 (publicado el 30 de octubre del año 2000) es un procedimiento dirigido a quienes estén dispuestos a someterse a una serie de evaluaciones con el objeto de acreditar los conocimientos que poseen.

${ }^{1}$ Resultados definitivos del XIII Censo General de Población y Vivienda 2000., INEGI

${ }^{2}$ Anuario Estadístico de la ANUIES., 2000. 
La Secretaría de Educación Pública, por medio de la Dirección General de Acreditación, Incorporación y Revalidación se encarga de analizar las solicitudes. El proceso de evaluación es llevado a cabo por alguna institución evaluadora designada por la SEP. Se trata de un programa novedoso donde el interesado se somete a una serie de exámenes para alcanzar la acreditación. Es un proceso rigorista y serio basado, sustancialmente, en los conocimientos y habilidades que demuestre el sustentante en las evaluaciones.

\section{ANTECEDENTES LEGALES DEL ACUERDO 286}

El primer antecedente legal aparece en la Ley Federal de Educación de 1973, que en su artículo 66 plantea:

"La Secretaría de Educación Pública creará un sistema federal de certificación de conocimientos, por medio del cual se expedirán certificados de estudios y se otorgará diploma, título o grado académico que acredite el saber demostrado, de acuerdo con el Reglamento que al efecto se expida..." ${ }^{3}$.

El reglamento, desafortunadamente, nunca se expidió; por lo tanto, no se aplicó este artículo.

Por su parte, la Ley General de Educación (publicada en el Diario Oficial, el 13 de julio de 1993), manifiesta lo siguiente:

"Artículo 64. La Secretaría, por acuerdo de su titular, podrá establecer procedimientos por medio de los cuales se expedirán certificados, constancias, diplomas o títulos a quienes acrediten conocimientos terminales que correspondan a cierto nivel educativo o grado escolar, adquiridos en forma autodidacta o a través de la experiencia laboral.

„El acuerdo secretarial respectivo señalará los requisitos específicos que deban cumplirse para la acreditación de conocimientos" ${ }^{4}$.

\footnotetext{
${ }^{3}$ Ley General de Educación., 1973.

${ }^{4}$ FUENTE: [http://www.sep.gob.mx/wb2/sep/sep_2256_ley_general_de_educa]., página 19., consulta el 13 de junio de 2003.
} 
En 1998, la Escuela Superior de Administración de Instituciones (ESDAI), perteneciente a la Universidad Panamericana, solicitó a la SEP, sobre la base del artículo 64 de la Ley General de Educación, que sus egresadas tituladas de las generaciones de 1964 a 1993, pudieran obtener el título autenticado por la Secretaría. La SEP aceptó, pero con la condición de que aprobaran un examen diseñado por el CENEVAL. Tras ello, se firmó el convenio en noviembre de 1998; y de enero de 1999 a agosto de 2000, se desarrollaron los instrumentos de evaluación.

La SEP autorizó a 216 egresadas para que participaran en este proceso. 191 egresadas se sometieron a examen, de las cuales 161 cumplieron con los criterios de acreditación establecidos y lograron autenticar su título y obtener su cédula profesional.

Lo anterior constituyó la antesala para la creación y aplicación del Acuerdo secretarial número 286, publicado en el DOF, el 30 de octubre de 2000.

\section{FUNDAMENTOS LEGALES DEL ACUERDO 286}

El Acuerdo 286 está definido de la siguiente manera:

"Acuerdo 286 por el que se establecen los lineamientos que determinan las normas y criterios generales, a que se ajustarán la revalidación de estudios realizados en el extranjero y la equivalencia de estudios, así como los procedimientos por medio de los cuales se acreditarán conocimientos correspondientes a niveles educativos o grados escolares adquiridos en forma autodidacta, a través de la experiencia laboral o con base en el régimen de certificación referido a la formación para el trabajo" ${ }^{5}$.

Los fundamentos legales que soportan al anterior Acuerdo son:

Diario Oficial de la Federación., 30 de octubre de 2000., primera sección., página 12 . 
- El artículo $3^{\circ}$ de la Constitución Política de los Estados Unidos Mexicanos, cuyos preceptos orientan y obligan al Estado mexicano a procurar que los individuos se desarrollen armónicamente, mejoren económica, social y culturalmente; así como a defender, preservar y acrecentar nuestra cultura, la independencia económica y política.

- 38 fracción XV de la Ley Orgánica de la Administración Pública Federal. Dicho ordenamiento indica que a la Secretaría de Educación Pública le corresponden los asuntos de: "Revalidar estudios y títulos, y conceder autorización para el ejercicio de las capacidades que acrediten" ${ }^{6}$.

- 11, 12 fracción VIII, 13 fracción V, 14 fracción III, 43, 44, 45, 60, 61, 62, 63 y 64 de la Ley General de Educación.

El artículo 11 expone la responsabilidad que las autoridades educativas tienen de aplicar y vigilar el cumplimiento de la Ley General de Educación. Por su parte los artículos 12, 13 y 14 , hacen referencia a la regulación del sistema nacional de créditos, de revalidación y de equivalencias, que se efectuarán por medio de los lineamientos generales que la propia Secretaría expida; a fin de facilitar el tránsito de educandos de un tipo o modalidad educativo a otro y otorgar las equivalencias de estudios y revalidaciones a lugar ${ }^{7}$.

Los artículos 43, 44 y 45 se enfocan a la educación para adultos (comprendiendo desde la alfabetización, la educación primaria y la secundaria, así como la formación para el trabajo), y manifiestan que la acreditación de los conocimientos podrá realizarse mediante exámenes parciales o globales y que, en caso de no obtenerse un resultado, tendrán derecho

${ }^{6}$ FUENTE: [http://wwwsecodam.gob.mx/leyes/loapf2.htm]., páginas 23 y 24., consulta del 8 de mayo de 2003.

7 FUENTE: [http://www.sep.gob.mx/wb2/sep/sep_2256_ley_general_de_educa]., páginas 3 y 4., consulta del 13 de junio de 2003. 
a presentar nuevos exámenes hasta obtener la acreditación de dichos conocimientos ${ }^{8}$.

En los artículos 60 al 64 de la anteriormente citada Ley General de Educación, se manifiesta que la legalidad de los estudios realizados dentro del sistema educativo nacional, así como los estudios revalidados, tienen validez oficial en toda la República. Se estipula, además, que es la Secretaría de Educación Pública quien dicta las normas y criterios generales a los que se ajustarán la revalidación y la declaración de estudios equivalentes. En ese mismo tenor, se establece que quedará bajo la determinación de la SEP el instaurar los procedimientos por medio de los cuales se expedirán certificados, constancias, diplomas o títulos a aquellos que acrediten conocimientos, habilidades y destrezas que se correspondan a cierto nivel educativo o grado escolar, adquiridos en forma autodidacta o a través de la experiencia laboral ${ }^{9}$.

- 5o Fracción XVI, 18 fracciones I, VIII y XVII del Reglamento Interior de la Secretaría de Educación Pública.

En los dos artículos mencionados del Reglamento Interior de la SEP se fijan los lineamientos de carácter general que la Ley General de Educación atribuye a la Secretaría de Educación Pública -referente a la planeación, programación y coordinación-, así como el desarrollo de las actividades y eventos que le corresponda instrumentar a SEP, en sus vínculos con los órganos legislativos ${ }^{10}$.

El Acuerdo 286 se integra por tres Títulos donde se especifican las disposiciones generales, normas y lineamientos del Acuerdo.

${ }^{8}$ FUENTE: [http://www.sep.gob.mx/wb2/sep/sep_2256_ley_general_de_educa]., página 9., consulta del 13 de junio de 2003.

${ }^{9}$ FUENTE: [http://www.sep.gob.mx/wb2/sep/sep_2256_ley_general_de_educa]., p. 12., consulta del 13 de junio de 2003.

${ }^{10}$ FUENTE: [http://www.sep.gob.mx/web2/sep/sep_Capitulo_VI]., consulta del 13 de junio de 2003 . 
Su Título Primero manifiesta:

"El presente Acuerdo tiene por objeto fijar los lineamientos que establecen las normas y criterios generales, aplicables a toda la República, a que se ajustarán la revalidación de estudios realizados en el extranjero y la declaración de estudios equivalentes. Asimismo, establecer los procedimientos y requisitos específicos a que deberán de ajustarse los particulares para acreditar conocimientos correspondientes a ciertos niveles educativos o grados escolares adquiridos en forma autodidacta, a través de la experiencia laboral o con base en el régimen de certificación referido a la formación para el trabajo" ${ }^{11}$.

Lo anterior hace referencia a las etapas y trámites que deben seguirse a fin de llevar a cabo los actos administrativos a través de los cuales se declaran equiparables entre sí, estudios efectuados dentro del sistema educativo nacional, el otorgamiento de validez oficial a estudios efectuados fuera del sistema educativo nacional, así como los procedimientos y requisitos que deben seguir los particulares a fin de obtener la acreditación de conocimientos que se corresponden a cierto nivel educativo, adquiridos en forma autodidacta $\mathrm{y} / \mathrm{o}$ vía de la experiencia laboral.

Por su parte, el Título Segundo señala los:

"Lineamientos que determinan las normas y criterios generales, a que se ajustarán la revalidación de estudios realizados en el extranjero y la equivalencia de estudios" ${ }^{12}$.

Es, por tanto, el Título Segundo donde se especifican las características, condiciones y procedimientos que se requieren cumplir a fin de obtener la revalidación o la equivalencia de estudios.

${ }^{11}$ Diario Oficial de la Federación., 30 de octubre de 2000., página 12.

${ }^{12}$ Diario Oficial de la Federación., 30 de octubre de 2000., página 14. 
Lo que a continuación se plasma hace referencia a la Educación Superior, aunque es importante recalcar que existen disposiciones específicas para la Educación Primaria y Secundaria, para la Educación Media Superior; así como para la Educación Normal.

En lo asentado en el Título Segundo, referente a las revalidaciones totales de tipo superior (exceptuando a la educación normal) se estipuló que para otorgar la revalidación total o por nivel completo de tipo superior, se requiere que los estudios acreditados y concluidos en el extranjero sean equiparables, al menos en un $75 \%$, a los estudios existentes dentro del sistema educativo nacional.

En lo concerniente a la revalidación parcial de tipo superior (exceptuando a la educación normal) se determinó que si del análisis de la solicitud de revalidación se desprende que los estudios no se equiparan (al menos en un $75 \%$ a los estudios existentes dentro del sistema educativo nacional), se otorgará revalidación parcial. Pudiéndose cursar, por parte del interesado, las asignaturas restantes en cualquier institución de educación superior pública o privada.

Acerca de la evaluación de conocimientos de tipo superior (excepto Educación Normal) se resolvió lo siguiente: El interesado con dictamen de revalidación parcial de tipo superior podrá optar por sujetarse a una evaluación global que le permita acreditar que posee los conocimientos suficientes para obtener una resolución de revalidación por nivel completo. Y de no acreditar la evaluación, podrá ser sujeto a una nueva y última evaluación global que, de no acreditarla, el interesado se sujetará a lo dispuesto en la resolución de revalidación parcial previamente obtenida (hasta el 30 de noviembre de 2004 se han otorgado 12 oficios de revalidación bajo esta modalidad). 
El Título Tercero hace referencia a:

"Procedimientos por medio de los cuales se acreditarán conocimientos que correspondan a un cierto nivel educativo o grado escolar, adquiridos en forma autodidacta, a través de la experiencia laboral o con base en el régimen de certificación referido a la formación para el trabajo" ${ }^{13}$.

Mediante este Título, el procedimiento general ha seguir, para la acreditación de conocimientos, los requisitos que deben cumplirse, los documentos que necesitan presentarse, las evaluaciones a efectuarse, entre otros puntos (como la improcedencia y revocación que pudiese proceder).

Lo anterior constituyó la base legal con la que surgió el Acuerdo 286, y que apareció publicado en el Diario Oficial de la Federación, con fecha del 30 de octubre de 2000, y en fecha 6 de noviembre del año 2000.

\section{REQUISITOS QUE DEBEN CUMPLIR LOS INTERESADOS}

Los interesados en acreditar conocimientos, conforme a los lineamientos que estipula el Acuerdo 286 -independientemente del nivel educativo que tengan- deberán cubrir, íntegramente, los siguientes requisitos:

- Tener 15 o más años de edad.

- Formular la solicitud correspondiente ante la autoridad educativa respectiva.

- Aprobar las evaluaciones con los puntajes que se determinen.

- Cubrir adicionales requisitos e información, de acuerdo a las disposiciones que resulten aplicables.

${ }^{13}$ Diario Oficial de la Federación., 30 de octubre de 2000., página 23. 
Los anteriores requerimientos son generales para todos los niveles educativos, además de ellos, existen obligaciones adicionales según las convocatorias concretas.

Para el nivel superior los requisitos son:

- Tener 30 años o más.

- Contar con cinco años de experiencia laboral en la licenciatura que se desea acreditar.

- En caso de tener estudios formales en la licenciatura que se pretende acreditar, se deberán tener cinco años o más de haber interrumpido los estudios profesionales.

- Ser de nacionalidad mexicana.

- Presentar solicitud por escrito, en el formato que será entregado por la Secretaría de Educación Pública.

Acompañarán la solicitud, los siguientes documentos:

- Original y copia del acta de nacimiento.

- Original y copia del certificado de bachillerato.

- Currículum vitae sintético con soporte documental.

- Original y copia de una identificación oficial con fotografía.

Si el interesado cumple con los anteriores requisitos se le otorga un oficio, denominado "acuerdo de admisión", donde se estipula que puede iniciar su proceso de evaluación.

Es importante mencionar que el 30 de julio de 2003, en el Diario Oficial de la Federación, se publicó el Acuerdo número 328 , el cual modifica el diverso Acuerdo 286. Entre las modificaciones fundamentales se halla la exigencia de cubrir, por lo menos, el porcentaje de créditos según el perfil profesional que se marca en la siguiente tabla: 
TABLA 1

\begin{tabular}{|l|l|l|}
\hline \multicolumn{1}{|c|}{$\mathbf{5 0 \%}$} & \multicolumn{1}{|c|}{$\mathbf{6 0 \%}$} & \multicolumn{1}{c|}{$\mathbf{7 0 \%}$} \\
\hline Comercio y Negocios & Administración & Actuaría \\
Internacionales & Informática & Ciencias Farmacéuticas \\
Mercadotecnia & Psicología & OFB \\
Pedagogía & & Ingeniería Agronómica \\
Ciencias de la Educación & & Ingeniería Civil \\
Turismo & & Ingeniería Eléctrica \\
& & Ingeniería electrónica \\
& & Ingeniería Industrial \\
& & Ingeniería Mecánica \\
& & Ingeniería Mecánica y \\
& & Eléctrica \\
& & Ingeniería Química \\
& & Medicina General \\
& & Odontología \\
& & Enfermería \\
& & Derecho \\
& & Contaduría \\
& & Médico Veterinario y \\
& & Zootecnista \\
\hline
\end{tabular}

En caso de que el interesado no cubra el porcentaje establecido o que haya adquirido conocimientos en forma autodidacta -con excepción de las áreas de la salud- podrá acceder al proceso de evaluación siempre y cuando el Colegio de profesionistas del área emita una opinión favorable a fin de iniciar el proceso de evaluación ${ }^{14}$.

El proceso de evaluación lo lleva a cabo la entidad designada por la SEP e integra distintas evaluaciones globales; en caso de ser acreditadas satisfactoriamente, se procede a la expedición del título y cédula profesional respectiva por parte de la SEP.

${ }^{14}$ Diario Oficial de la Federación., 30 de julio de 2003., Segunda Sección., páginas 42-46. 


\section{PROCEDIMIENTO DEL ACUERDO 286 PARA EL NIVEL SUPERIOR}

Los pasos que debe realizar el interesado para intentar acreditar una determinada licenciatura conforme a los lineamientos del Acuerdo 286 son:

- Consultar los requisitos y características del procedimiento de acreditación.

- Formular una solicitud ante la autoridad educativa respectiva (pudiendo ser la DGAIR o la representación estatal de la SEP en la localidad).

La autoridad educativa evaluará la solicitud y, si es el caso, otorgará un acuerdo de admisión del trámite e informará al interesado y a la institución evaluadora. El interesado se presentará en la institución evaluadora a fin de recibir orientación sobre el proceso. Esta institución aplicará las evaluaciones pertinentes y remitirá el dictamen correspondiente a la autoridad educativa.

En caso de ser satisfactorio el resultado del proceso de evaluación, el interesado efectuará los trámites necesarios a fin de obtener el título y la cédula profesional respectiva ante la autoridad educativa.

\section{CONVENIO DE COLABORACIÓN SEP-CENEVAL, REFERENTE AL ACUERDO 286}

La Secretaría de Educación Pública firmó un convenio de colaboración con el Centro Nacional de Evaluación para la Educación Superior, A.C., a fin de coordinar esfuerzos para efectuar las evaluaciones de los niveles medio superior y superior referidas en el Acuerdo 286, a partir de ese momento (entre los meses de diciembre de 2000 y febrero de 2001), los diversos Consejos Técnicos de los EGEL del CENEVAL se dedicaron a trabajar a fin de diseñar los procedimientos de evaluación correspondientes iniciándose, en diciembre de 2000, la atención de sustentantes en un nuevo departamento dentro del CENEVAL. 


\section{INSTRUMENTACIÓN DE LOS PROCESOS DE EVALUACIÓN CONFORME AL ACUERDO 286}

A solicitud de la SEP y de forma piloto, se realizó un primer examen de administración, a fin de identificar si el EGEL y una entrevista oral sobre la actividad laboral eran los únicos elementos a tomar en cuenta en el proceso de evaluación. El examen escrito se realizó el día 24 y el oral el 27 de noviembre de 2000, resultando aprobado el sustentante.

Ante esta experiencia se decidió diseñar los procedimientos de evaluación para todas las licenciaturas que el CENEVAL acreditaría con base al convenio firmado. Dichos procesos quedaron conformados con una serie de evaluaciones (teóricas, orales y/o prácticas).

Las carreras que actualmente se acreditan dentro del Acuerdo 286 son:

1. Administración.

2. Ciencias de la Educación.

3. Ciencias Farmacéuticas.

4. Comercio y Negocios Internacionales.

5. Contaduría.

6. Derecho.

7. Enfermería Licenciatura.

8. Enfermería Técnico Medio Superior.

9. Informática y Computación.

10. Ingeniería Agronómica.

11. Ingeniería Civil.

12. Ingeniería Eléctrica.

13. Ingeniería Electrónica.

14. Ingeniería Industrial.

15. Ingeniería Mecánica.

16. Ingeniería Mecánica-Eléctrica.

17. Ingeniería Química.

18. Medicina General.

19. Medicina Veterinaria y Zootecnia.

20. Mercadotecnia. 
21. Odontología.

22. Pedagogía.

23. Psicología.

24. Turismo.

En lo general, el proceso implica obtener el acuerdo de admisión por parte de la SEP (DGAIR), a fin de entablar contacto con la institución evaluadora. En caso de ser el CENEVAL, el sustentante deberá seguir los siguientes pasos:

- Asistir a una sesión de orientación donde se le expondrán las condiciones y características del proceso de evaluación.

- Registrarse y sustentar el correspondiente Examen General de Egreso de la Licenciatura (EGEL).

- Entregar una serie de documentos (desde el currículum y la correspondiente documentación que lo soporte, hasta la presentación de un trabajo escrito).

- Sustentar una evaluación ante sinodales, consistente en una entrevista sobre su desarrollo y experiencia laboral, la exposición y réplica del trabajo escrito y la presentación de un examen oral. Es importante señalar que en los exámenes de las áreas de la salud y de las ingenierías y tecnologías, se requiere sustentar por lo menos un examen práctico, clínico o teórico-práctico.

En caso de obtener un resultado aprobatorio en el proceso de evaluación, el interesado tramitará, ante la SEP, la expedición del título y cédula profesional respectiva.

A continuación, se presentan cuatro ejemplos de los procedimientos que deben llevar a cabo los interesados en acreditar alguna licenciatura por medio del Acuerdo 286, definidos y autorizados por los Consejos Técnicos de los EGEL del CENEVAL: 
TABLA 2

\begin{tabular}{|c|c|}
\hline ÁREA & $\begin{array}{l}\text { CARACTERÍSTICAS } \\
\text { GENERALES DEL PROCESO } \\
\text { DE EVALUACIÓN }\end{array}$ \\
\hline $\begin{array}{l}\text { Ciencias Sociales, } \\
\text { Humanidades y } \\
\text { Económico Administrativas }\end{array}$ & $\begin{array}{l}\text { Sustentación del Examen General } \\
\text { para el Egreso de la Licenciatura. } \\
\text { Elaboración de un trabajo por escrito. } \\
\text { Entrevista ante sinodales. } \\
\text { Sustentación de un examen oral } \\
\text { ante sinodales. }\end{array}$ \\
\hline
\end{tabular}

\begin{tabular}{|l|l|}
\hline ÁREA & CARACTERÍSTICAS GENERALES \\
& DEL PROCESO DE EVALUACIÓN \\
\hline Ciencias de la Salud & Sustentación del Examen General para \\
& el Egreso de la Licenciatura. \\
& Entrevista ante sinodales. \\
& Sustentación de un examen oral ante \\
& sinodales. \\
& Elaboración de un trabajo por escrito. \\
& Sustentación de un examen práctico \\
& ante sinodales. \\
\hline
\end{tabular}

\begin{tabular}{|c|c|}
\hline ÁREA & $\begin{array}{l}\text { CARACTERÍSTICAS GENERALES DEL } \\
\text { PROCESO DE EVALUACIÓN }\end{array}$ \\
\hline Ingenierías y Tecnológicas & $\begin{array}{l}\text { Sustentación del Examen General para el } \\
\text { Egreso de la Licenciatura. } \\
\text { Entrevista ante sinodales. } \\
\text { Elaboración de un trabajo por escrito. } \\
\text { Sustentación de un examen teórico-práctico } \\
\text { ante sinodales. }\end{array}$ \\
\hline
\end{tabular}

Para el desarrollo de cada proceso de evaluación, se decidió proponer un proceso sencillo en su lógica pero que cumpliera la necesidad de evaluar pertinentemente conocimientos, habilidades, destrezas, actitudes y valores. 


\section{INSTRUMENTACIÓN DE LOS PROCESOS DE EVALUACIÓN CONFORME AL ACUERDO 286}

Los procedimientos de evaluación aplicables al Acuerdo 286 para el nivel de licenciatura, dan inicio con la aplicación del EGEL correspondiente; dichos exámenes fueron desarrollados por Consejos Técnicos, integrados por profesionales y representantes de las instituciones educativas de nivel superior más importantes del país, a fin de contar con los conocimientos de expertos involucrados en la formación profesional de los licenciados de dichas licenciaturas.

Las actividades realizadas en los Consejos han tenido como fin el diseño y la definición de criterios, indicadores, estándares y procedimientos a seguir en cada proceso de evaluación para la acreditación de conocimientos de la licenciatura respectiva, conforme a los lineamientos que se establecen en el Acuerdo 286 de la Secretaría de Educación Pública.

El análisis de los planes y programas de estudio vigentes que ofrecen las diversas instituciones educativas, así como la determinación de los elementos indispensables, características, actitudes, valores, destrezas y conocimientos con que debe contar un egresado de determinada licenciatura, fueron actividades fundamentales realizadas por los Consejos Técnicos a fin de determinar el mecanismo que apreciara, de la mejor manera posible, el grado en que un sustentante satisface los requerimientos definidos y aceptados para lograr la acreditación.

Los exámenes se estructuran sobre la base de los planes y programas de estudio vigentes en el ámbito nacional, organizados en áreas específicas para determinar los conocimientos y habilidades que todo egresado del nivel licenciatura debe poseer al término de sus estudios profesionales.

Los Consejos Técnicos han desarrollado una "guía de estudio", específica para cada una de las licenciaturas, diseñadas para orientar, aconsejar y asesorar al sustentante en todo aquello que contribuya a lograr su óptimo desempeño en el examen. Incluye información respecto a la estructura y 
composición del examen; las áreas, temas y subtemas que lo integran; indicaciones y consejos para presentarlo, así como ejemplos de preguntas parecidas a las que lo conforman.

La utilización de la guía permite al sustentante disminuir las probabilidades de incurrir en equivocaciones y errores de interpretación o apreciación mientras resuelve el examen, ayudando con ello a aprovechar su máxima capacidad en la demostración del nivel de posesión de los conocimientos.

\section{POBLACIÓN INSCRITA EN \\ EL PROCESO DE EVALUACIÓN}

La población atendida dentro del Acuerdo 286 es muy heterogénea. Son muchas las razones para explicar dicha diversidad: desde el factor económico hasta el nivel educativo alcanzado por los sustentantes.

La diversidad de la población atendida dentro del Acuerdo 286, da muestras del deseo de ampliar las opciones educativas que guían al proceso. El posibilitar el cauce de las aspiraciones y exigencias de la sociedad, permite incrementar la inteligencia colectiva del país, eleva la capacidad de la población para desempeñarse mejor en el ámbito laboral, lo que a su vez redunda en el desarrollo nacional.

La siguiente tabla muestra la distribución de los sustentantes del Acuerdo 286 a nivel licenciatura, hasta el 30 de noviembre de 2004. La columna "Admitidos SEP", se refiere a aquéllos interesados en acreditar una determinada licenciatura y que han cubierto los prerrequisitos necesarios para iniciar el proceso de evaluación, además de haber obtenido el acuerdo de admisión otorgado por la SEP. Dentro del grupo de datos incluidos en "Presentación del EGEL" se exponen la totalidad de sustentantes que han presentado el EGEL. En las columnas designadas como "Suficientes" y "Sobresalientes" se muestran la totalidad de exámenes orales presentados por licenciatura y que han obtenido un dictamen aprobatorio en el proceso de evaluación. 
TABLA 3

\begin{tabular}{|c|c|c|c|c|}
\hline LICENCIATURA & $\begin{array}{l}\text { ADMITIDOS } \\
\text { SEP }\end{array}$ & $\begin{array}{l}\text { PRESENTACIÓN } \\
\text { DEL EGEL }\end{array}$ & SUFICIENTE & SOBRESALIENTE \\
\hline Actuaría & 19 & 0 & 0 & 0 \\
\hline Administración & 969 & 416 & 114 & 3 \\
\hline Ciencias Agronómicas & 101 & 22 & 0 & 0 \\
\hline Ciencias Farmacéuticas & 197 & 103 & 8 & 0 \\
\hline $\begin{array}{l}\text { Comercio y Negocios } \\
\text { Internacionales }\end{array}$ & 17 & 9 & 3 & 0 \\
\hline Contaduría & 923 & 389 & 60 & 1 \\
\hline Derecho & 1331 & 711 & 119 & 3 \\
\hline Informática-Computación & 515 & 205 & 23 & 1 \\
\hline Ingeniería Civil & 276 & 101 & 7 & 0 \\
\hline Ingeniería Eléctrica & 101 & 40 & 2 & 0 \\
\hline Ingeniería Electrónica & 131 & 52 & 2 & 0 \\
\hline Ingeniería Industrial & 256 & 116 & 7 & 0 \\
\hline Ingeniería Mecánica & 67 & 31 & 1 & 0 \\
\hline Ingeniería Mecánica-Eléctrica & 58 & 32 & 1 & 0 \\
\hline Ingeniería Química & 86 & 48 & 0 & 0 \\
\hline Licenciatura en Enfermería & 139 & 54 & 6 & 0 \\
\hline Medicina General & 202 & 133 & 1 & 0 \\
\hline $\begin{array}{l}\text { Medicina Veterinaria } \\
\text { y Zootécnia }\end{array}$ & 77 & 6 & 0 & 0 \\
\hline Mercadotecnia & 25 & 8 & 1 & 0 \\
\hline Odontología & 117 & 38 & 0 & 0 \\
\hline Pedagogía & 1793 & 560 & 108 & 0 \\
\hline Psicología & 378 & 148 & 54 & 11 \\
\hline Técnico en Enfermería & 383 & 70 & 9 & 0 \\
\hline Turismo (Gestión Empresarial) & 68 & 6 & 2 & 0 \\
\hline Turismo (Planeación y Desarrollo) & & 1 & 0 & 0 \\
\hline TOTAL & 8229 & 3299 & 528 & 19 \\
\hline
\end{tabular}

Los sustentantes del Acuerdo buscan concretar legítimas aspiraciones; el titularse representa no desperdiciar todos los esfuerzos y recursos familiares destinados a la consecución de los estudios que efectuaron. 


\section{EL VALOR DE LA PRÁCTICA Y LA AUTOFORMACIÓN}

Lo realmente relevante dentro del Acuerdo son los individuos detrás de los números presentados. Es significativa la propuesta pedagógica del Acuerdo 286 por la población a la que dirige su actividad, propuesta fundamentada en los aprendizajes basados en la actividad del sujeto que construye progresivamente el conocimiento.

En el proceso del Acuerdo, se hace referencia obligada a temas de equidad (oferta educativa y condiciones), calidad (en función de la satisfacción de los usuarios o beneficiarios del proceso), globalización (en función de nuevos propósitos educativos más relacionados con el logro de habilidades y competencias que permitan a cualquier sujeto, miembro de la sociedad, adaptarse o modificarla), entre otros.

Su puesta en marcha obliga a tomar conciencia que flexibilizar el sistema educativo es imperioso dada la necesidad de que las personas puedan adquirir e incorporar continuamente nuevos saberes; es decir, se ha hecho obligatorio generar y reconocer nuevas formas de aprendizaje, diferentes a los tradicionales modelos escolarizados. Posibilitar el reconocimiento de aquellas competencias profesionales, adquiridas en lugares ajenos a instituciones educativas, es un requisito vital en sociedades que poseen esquemas de producción que demandan una incorporación constante de nuevos saberes.

El Acuerdo 286 representa una innovación en los procesos de evaluación educativa y los proyectos pedagógicos del país e, incluso, en el ámbito mundial. Su importancia se manifiesta al ofrecer a las personas el poder hacerse acreedoras a un título o certificado.

La posibilidad de acreditar un determinado nivel educativo a través de procesos como los que marca el Acuerdo 286, supera ampliamente las expectativas planteadas originalmente. Lo cual no indica que sea una vía fácil o rápida de obtener grados académicos. Únicamente quienes poseen las actitudes, valores, destrezas y conocimientos que se 
corresponden a determinado nivel o perfil, se encuentran en posibilidad de acreditar los procesos de evaluación.

La implementación del Acuerdo 286 generó polémica en algunos sectores; polémica más política que académica. Si se confronta la formación académica con la formación práctica, nos hallaremos frente a elementos que no son antagónicos en su naturaleza, antes bien, son complementarios.

Los recintos educativos tienen como meta lograr los aprendizajes necesarios para el ejercicio de determinada profesión en el estudiantado -aprendizajes que van más lejos de los saberes teóricos y que son saberes susceptibles de adquirirse por medios no necesariamente escolares-. Por su parte, y de manera similar, la formación de los estudiantes en las instituciones de educación, se realiza en una buena medida enfrentándose al quehacer práctico, es decir: se aprende "haciendo".

Es claro que todo proceso emergente es factible de mejora. Esta visión es compartida por quienes participamos en la instrumentación del Acuerdo 286: el desarrollo de metodologías más funcionales es una búsqueda constante a fin de proporcionar servicio oportuno, ágil y eficiente a los sustentantes; así como para garantizar que quien ostente un título del Acuerdo 286 cumpla con el perfil idóneo de su disciplina.

El Acuerdo 286 ha permitido obtener o conservar empleos; ha favorecido expectativas personales y ha representado la satisfacción de muchas familias por la obtención de un título. 
REFERENCIAS BIBLIOGRÁFICAS

ANUIES., Anuario Estadístico de la ANUIES., México., 2000. BARTOLUCCI INCICO, Jorge., Desigualdad social, educación superior y sociología en México., México., Centro de Estudios Sobre la Universidad., UNAM., 1994., 154 págs.

CENEVAL., Acerca del CENEVAL y los Exámenes Generales para el Egreso de la Licenciatura., México., 1998., 132 págs. CENEVAl., Perfil del Ceneval., (Folleto)., México., 16 págs. CUELI, José (coordinador)., Valores y metas de la Educación en México., México., Ediciones de la Jornada., Serie Papeles de Educación., 1990., 165 págs.

Diario Oficial de la Federación., México., 30 de julio de 2003.

Diario Oficial de la Federación., México., 30 de octubre de 2000.

"Evaluación, acreditación y certificación", en Paedagogium, Revista Mexicana de Educación y Desarrollo., Año II., Número 4., Julio-diciembre 2001., México., 33 págs.

INEGI., Resultados Definitivos del XIII Censo General de Población y Vivienda 2000.,

MUÑOZ GARCÍA, Humberto., Los valores educativos y el empleo en México., México., Centro Regional de Investigaciones Multidisciplinarias e Instituto de Investigaciones Sociales., UNAM., 1996., 165 págs.

[http://www.geocities.com/Athens/Troy/8772/ley1973.htm]. [http://www.secodam.gob.mx/leyes/loapf2000.htm]. [http://www.secodam.gob.mx/leyes/loapf2000.htm]. http://www.sep.gob.mx/wb2/sep/sep 2256 ley general de educa]. [http://www.sep.gob.mx/wb2/sep/sep_2256 ley general_de_educa]. 